\title{
Hidden Markov Model-based Pedestrian Navigation System using MEMS Inertial Sensors
}

\author{
Yingjun Zhang ${ }^{1}$, Wen Liu $^{2}$, Xuefeng Yang ${ }^{1}$, Shengwei Xing ${ }^{1}$ \\ ${ }^{1}$ Navigation College, Dalian Maritime University, 116026, Dalian China \\ ${ }^{2}$ Navigation College, Dalian Maritime University, 116026, Dalian China, dmuliuwen@sina.com (corresponding author)
}

\begin{abstract}
In this paper, a foot-mounted pedestrian navigation system using MEMS inertial sensors is implemented, where the zero-velocity detection is abstracted into a hidden Markov model with 4 states and 15 observations. Moreover, an observations extraction algorithm has been developed to extract observations from sensor outputs; sample sets are used to train and optimize the model parameters by the Baum-Welch algorithm. Finally, a navigation system is developed, and the performance of the pedestrian navigation system is evaluated using indoor and outdoor field tests, and the results show that position error is less than $3 \%$ of total distance travelled.
\end{abstract}

Keywords: Pedestrian navigation system, hidden Markov model, zero velocity detection, inertial sensors.

\section{INTRODUCTION}

$\mathrm{G}$ PS IS AN IMPORTANT component in the positioning system and plays a key role in outdoor positioning. However, GPS continues to struggle indoors due to the failure of satellite signals to penetrate buildings [1]. Furthermore, recent developments in the field of smart mobile terminals have led to an increased interest in indoor positioning and navigation. In most recent studies, indoor positioning and navigation has been discussed in two different ways, one is Local Positioning System (LPS), and the other one is Pedestrian Dead Reckoning (PDR) [2]. Compared with LPS, PDR approach has a number of attractive features: autonomy, cost-effectiveness, no installing markers or instrumentation in advance [3]. Specifically, PDR is divided into stride and heading system (SHS) and inertial navigation system (INS) [4]. Pedestrian inertial navigation system which is based on MEMS inertial sensors has gradually become an indoor navigation solution due to its independence, portability, and low cost, and can be used in 3D navigation environment.

Pedestrian inertial navigation system widely adopts a system framework that is characterized by extended Kalman filtering and strapping of MEMS inertial sensors on insteps, which is proposed by Foxlin [5]. The main problem of the system is error accumulation caused by inertial sensor drift error [4], [6], [7]. A considerable amount of literature has been published on this problem. Researchers found that two feet touch the ground alternately and stay for a short time for approximately $0.3 \mathrm{~s}-1 \mathrm{~s}$ in a normal walking cycle. The short interval is called the zero velocity intervals [8]. As the real velocity of foot in zero velocity intervals is zero, if a zero velocity state is detected, the velocity error can be eliminated. This algorithm is called zero velocity updates algorithm. Therefore, zero velocity detection is an extremely important part of the pedestrian navigation system. It provides the required information to reset the velocity error.

Zero velocity detection algorithms can be divided into two kinds. One option is based on the hypothesis that the measured acceleration is constant and equal to gravity and the measured angular velocity is zero in zero velocity intervals. A range of zero velocity detection algorithms have been proposed based on inertial sensor outputs, where the main difference is the disposal method of sensor outputs and the compound modes of inertial sensors. In reference [5], the zero velocity intervals are determined based on inertial sensors (accelerometers and gyroscopes) output norms. If the outputs are smaller than some thresholds for a predetermined time, then the zero velocity is decided. In reference [9], the zero velocity is determined based on $\mathrm{Z}$-axis accelerometer and $\mathrm{Y}$-axis gyroscope outputs. In reference [10], the zero velocity is determined based on gyroscope output norms. In reference [11], [12], the zero velocity intervals are determined based on the variance of accelerometer values. In reference [13], the zero velocity is determined based on norms of accelerometers and gyroscopes along with variance of accelerations. In reference [14], the zero-velocity detection problem is abstracted as a hypothesis-testing problem. However, the proposed algorithms need corresponding threshold values, if the chosen threshold values are too small or large, the real zero velocity intervals will be missed and the wrong interval will be detected. The other option is using the walking characteristics to detect the zero velocity intervals. Typically, the walking motion is modeled as a repeating sequence of push off, swing, heel strike and stance. If each state is detected accurately, then zero velocity intervals can be determined reliably.

Compared with the former algorithm which needs corresponding threshold values, the latter algorithm has a threefold advantage. To begin with, the latter algorithm is more reliable, because the threshold is constant, it may be suitable in some cases, but may be too small or large in some other cases. Secondly, the latter algorithm is based on the walking characteristics, not only the zero velocity intervals, but also the other stage can be determined, and the other stage is useful to verify the zero velocity intervals and inspire potential applications. Finally, the latter algorithm is a new research field which relates to pattern recognition, etc., therefore, the pedestrian navigation system will be extended with latter algorithm. 
The aim of this paper is to design a pedestrian inertial navigation system using MEMS inertial sensors which is based on the hidden Markov model. Specifically, the zero velocity detection problems are abstracted as a hidden Markov model. The observations extraction algorithm is developed to extract observations from sensor outputs, in addition, a sample set and Baum-Welch algorithm are used to train and optimize the model. Finally, the performance of the HMM-based pedestrian navigation system is evaluated using indoor and outdoor field tests, and the results show that the proposed algorithm is reliable.

The most relevant result is reference [8], where a zero velocity detection algorithm using a hidden Markov model is also used. The main difference is threefold. One is that statistical method is used in this paper to generate the initial observation probabilities while threshold values are used in reference [8]. The second difference is that a new observations extraction algorithm is developed. The data volume is only $5 \%$ of sensor outputs. The third difference is that the Baum-Welch algorithm is used in this paper to train and validate the hidden Markov model.

The paper has been organized in the following way. Section 2 explores characteristics of pedestrians through experiments. Section 3 abstracts the zero velocity detection into a hidden Markov model problem. Section 4 describes the pedestrian navigation system framework. Section 5 evaluates the performance of the HMM-based pedestrian navigation system using indoor and outdoor field tests.

\section{CHARACTERISTICS OF PEDESTRIANS WALKING MOTION}

Compared with traditional carriers of inertial system, such as aircrafts, ships and guided missiles, pedestrians are characterized by low speed, little mass, small inertial and periodic motion [15].

For the pedestrian walking motion, the movement depends on two feet. To be exact, two feet swing forward alternately, and the center of gravity of a pedestrian is moving horizontally with the rise and fall of a small scale. Two phases exist, one phase where the foot is firmly planted on the ground is called stance phase, and the foot is called supportive foot, providing a pivot point over which to vault. The other phase where a foot lifts from behind the pedestrian and swings to enter its stance phase is called swing phase, and the foot is called swing foot which breaks the fall. Therefore, the pedestrian walking motion can be characterized by alternate 'vaulting' of the body over a stiffened leg, with the fall being broken by the opposing leg [4].

In order to explore the motion characteristics of a pedestrian, inertial sensors are attached to the insteps to record inertial measurements at 120 samples per second. The Inertial Measurement Unit used in this work is the Xsens MTx sensor (model 28A53G25) (Xsens), as shown in Fig.1., which is the standard model with the accelerometers with a full scale of $\pm 50 \mathrm{~m} / \mathrm{s}^{2}$ and the gyroscopes with a full scale of $\pm 1200^{\circ} / \mathrm{s}$. It has a size of $38 \times 53 \times 21 \mathrm{~mm}$, and a weight of 30 grams. Therefore, it is small enough to be mounted on the instep of a pedestrian.
The inertial sensors outputs are collected by a laptop which is connected to the experimenter through serial port. The change rules of acceleration and angular velocity of the walking motion are shown in Fig.2.

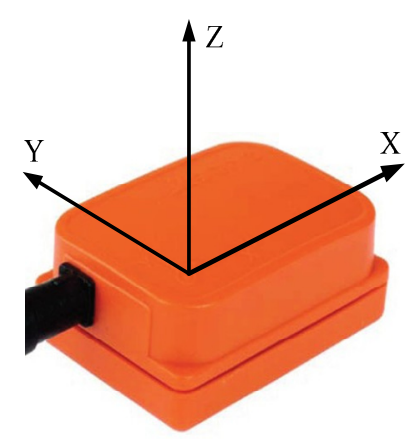

Fig.1. The MEMS inertial sensors MTX.

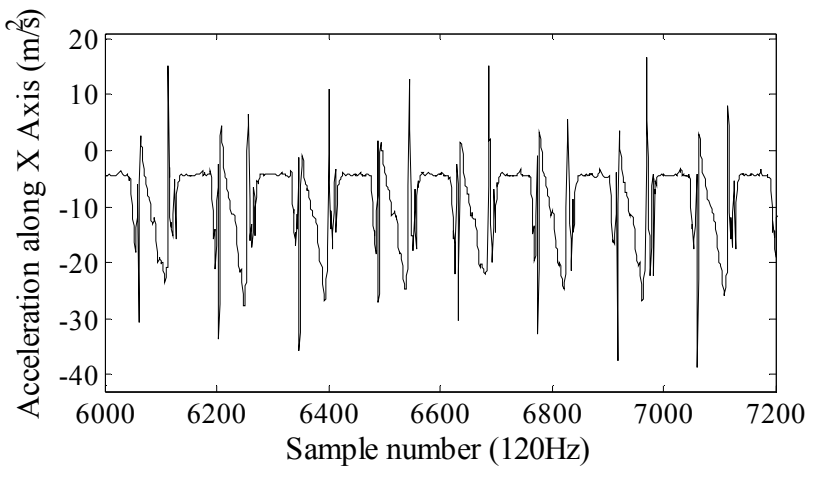

a) Acceleration along $X$ axis

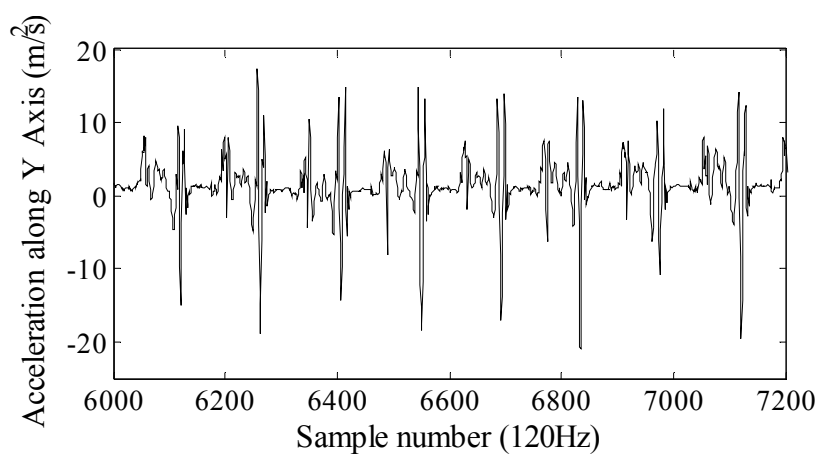

b) Acceleration along $\mathrm{Y}$ axis

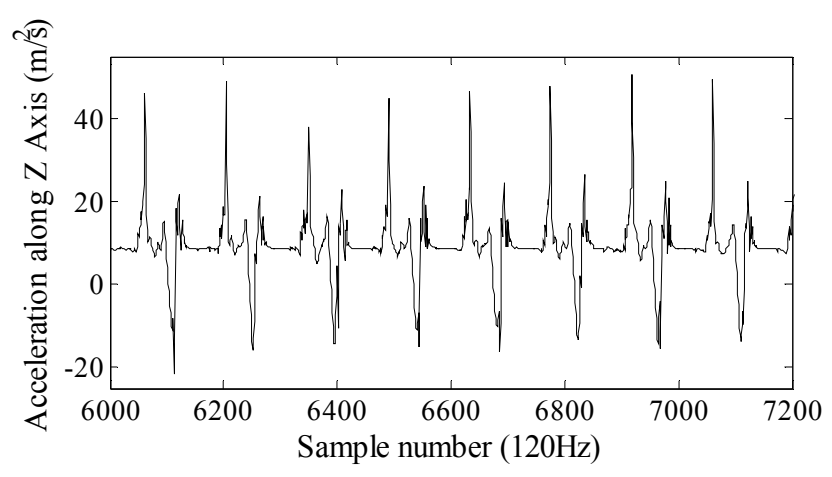

c) Acceleration along $\mathrm{Z}$ axis 


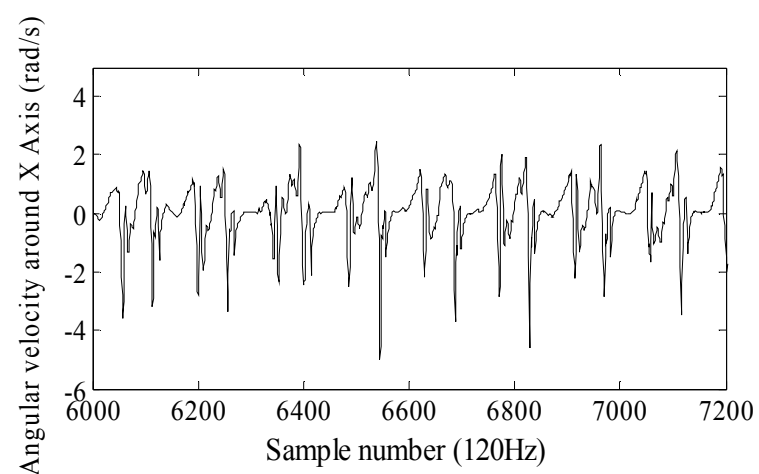

d) Angular velocity around $X$ axis

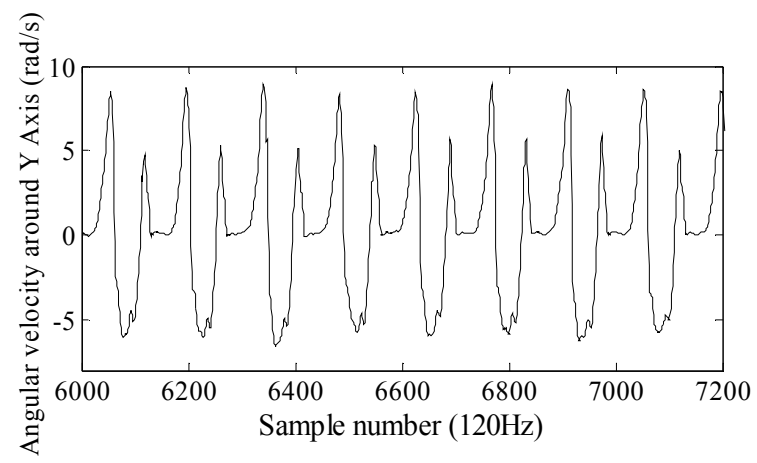

e) Angular velocity around $Y$ axis

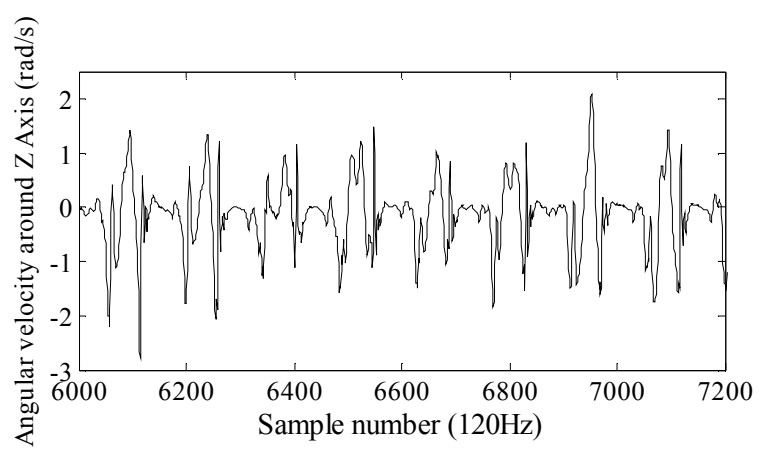

f) Angular velocity around $\mathrm{Y}$ axis

Fig.2. The acceleration and angular velocity curves during walking motion.

The data described above indicate that the acceleration changes periodically, especially, the acceleration along the $X$ axis changes between $15 \mathrm{~m} / \mathrm{s}^{2}$ and $-35 \mathrm{~m} / \mathrm{s}^{2}$, and the acceleration along the $Z$ axis changes between $-20 \mathrm{~m} / \mathrm{s}^{2}$ and $50 \mathrm{~m} / \mathrm{s}^{2}$. Similarly, the angular velocity around the three axes changes periodically. Compared with $\mathrm{X}$ and $\mathrm{Z}$ axes, the angular rate around the $\mathrm{Y}$ axis changes significantly, specifically, between $-7 \mathrm{rad} / \mathrm{s}$ and $9 \mathrm{rad} / \mathrm{s}$. What is more, the periodical characteristics are more obvious.

\section{THE ZERO VELOCITY DETECTION ALGORITHM}

This section studies how to describe the zero velocity detection problems with a hidden Markov model, such as description of observations, development of observations extraction algorithm to extract observations from sensors outputs, initial and train state transfer probability and observation probability. The hidden Markov model is a statistical Markov model on which the system being modeled is assumed to be a Markov process with unobserved states. The state is not directly visible, but output, dependent on the state, is visible. Each state has a probability distribution over the possible output. For pedestrian walking motion zero velocity detection, two random processes exist. One is measured acceleration and angular velocity with inertial sensors, which is a visible process. The other is transfer process of four states of walking motion, which is not visible.

Hidden Markov model is a probability model used to represent the statistic property of the stochastic process and is characterized by model parameters. In order to define an HMM completely, following elements are needed [16]:

(1) $\mathrm{N}$, the number of states of the model;

(2) $\mathrm{M}$, the number of mixtures in each state;

(3) $A=\left\{a_{i j}\right\}$, the state transition probability matrix

$$
a_{i j}=P\left\{q_{t+1}=j \mid q_{t}=i\right\} 1 \leq i, j \leq N
$$

Where $q_{t}$ is the state at time $\mathrm{t}$ and $a_{i j}$ is the transition probability from state $i$ to state $j$;

(4) $B=\left\{b_{j}\left(O_{t}\right)\right\}$, the output probability distribution where $b_{j}\left(O_{t}\right)$ is a finite mixture of Gaussian distributions associated with state $j$ of the form:

$$
b_{j}\left(O_{t}\right)=\sum_{m=1}^{M} c_{j m} G\left(\mu_{j m}, \sum_{j m}, O_{t}\right)
$$

Where $O_{t}$ is the $\mathrm{t}$-th observation vector, $c_{j m}$ is weighting coefficient for the $\mathrm{m}$-th mixture in state $j$, and $\mathrm{G}$ is the Gaussian distribution with mean vector $\mu_{j m}$ and covariance matrix $\sum_{j m}$ for the m-th mixture component in state $j$.

(5) $\pi=\left\{\pi_{j}\right\}$, the initial state distribution that is used to describe the probability distribution of the observation symbol in the initial moment when $\mathrm{t}=1$.

In order to abstract the zero velocity detection into a hidden Markov model, the states and observations are defined, and the state transition probability is initialized, in addition, the observation probability is initialized using the statistical analysis method, finally, the estimated state transition and observation probabilities are optimized using the Baum-Welch algorithm.

\subsection{States and observations}

Four states alternate in turn in a walking cycle, as is shown in Fig.3. The push off state is defined as state A, swing state is defined as state $B$, heel strike state is state $\mathrm{C}$ and stance state is state $\mathrm{D}$. As angular velocity around $\mathrm{Y}$ axis changes significantly (see Fig.2.e)), so it is chosen as observations. Fig.4. shows angular velocity changes during one walking cycle, and it is divided into four parts according to four states. As mentioned above, $\mathrm{Y}$ axis angular velocity is used 
to construct observations. Each state will have a different value in different cycle due to the randomness of walking motion. In order to describe each state, angular velocity is subdivided into 15 grades (see Fig.4.). So, 4 states (A, B, C and D) and 15 observations $(1,2,3,4,5,6,7,8,9,10,11$, $12,13,14$ and 15) are defined.

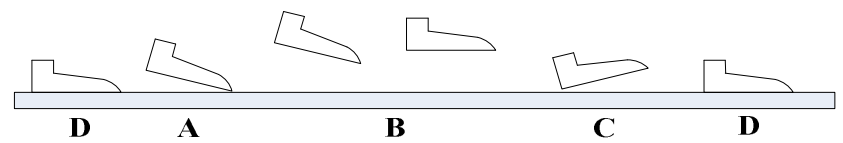

Fig.3. The state transition during a walking motion cycle.

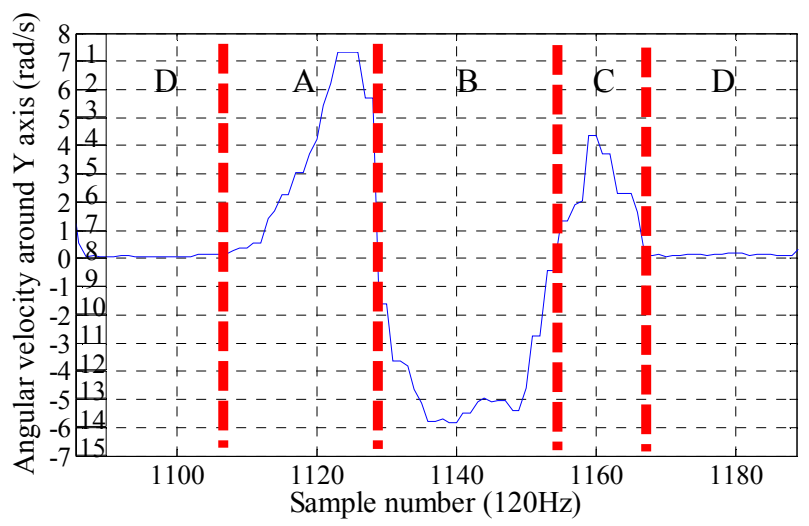

Fig.4. The definition of states and observations of HMM.

\subsection{State transition probability}

In pedestrian walking motion, the ideal state transition is $\mathrm{A} \rightarrow \mathrm{B} \rightarrow \mathrm{C} \rightarrow \mathrm{D} \rightarrow \mathrm{A}$, so the ideal state transition probability is A.

$$
A=\left[\begin{array}{llll}
0 & 1 & 0 & 0 \\
0 & 0 & 1 & 0 \\
0 & 0 & 0 & 1 \\
1 & 0 & 0 & 0
\end{array}\right]
$$

However, considering randomness motion and external factors, state transition is not the same as the ideal one. Therefore, parameters in A should be adjusted to cope with special state transitions. And $A_{1}$ is constructed to be the initial state transition probability, which is determined using the trial and error process.

$$
A_{1}=\left[\begin{array}{llll}
0.05 & 0.85 & 0.05 & 0.05 \\
0.05 & 0.05 & 0.85 & 0.05 \\
0.05 & 0.05 & 0.05 & 0.85 \\
0.85 & 0.05 & 0.05 & 0.05
\end{array}\right]
$$

In the following steps, the initial state transition probability will be optimized to get the final state transition probability.

\subsection{Observation probability}

Observation probability indicates the probability of each state generating each observation. In order to get initial observation probability, the statistical analysis method is used to process the observations in the sample set. The sample set is raw angular velocity measured by MEMS inertial gyroscopes. In addition, the observations extraction algorithm is developed to extract observations from sample set. The flow chart is shown in Fig.5.

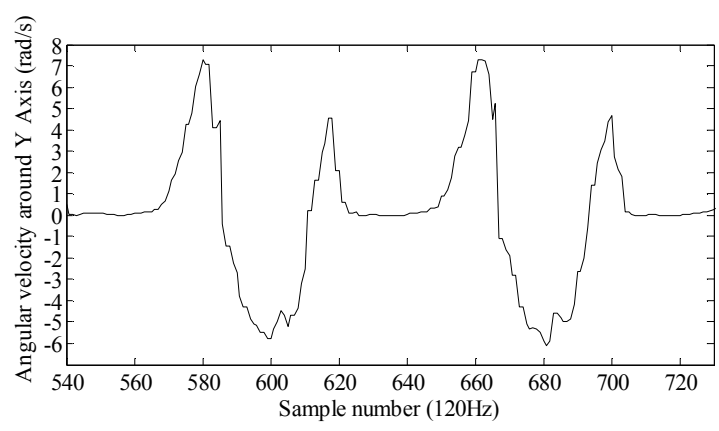

a) Raw angular velocity around $\mathrm{Y}$ axis.

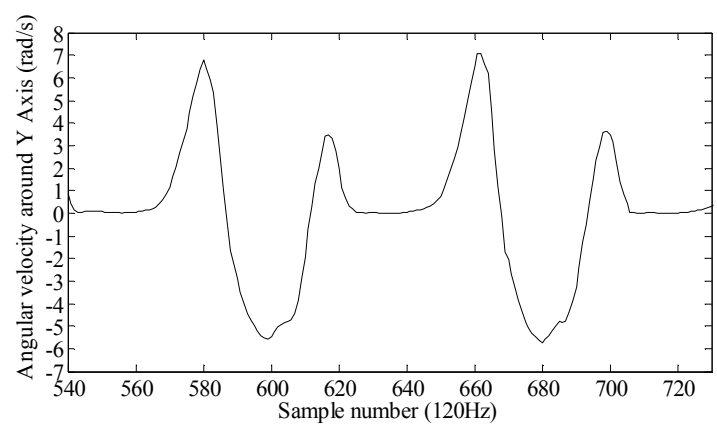

b) Smoothed angular velocity around $\mathrm{Y}$ axis

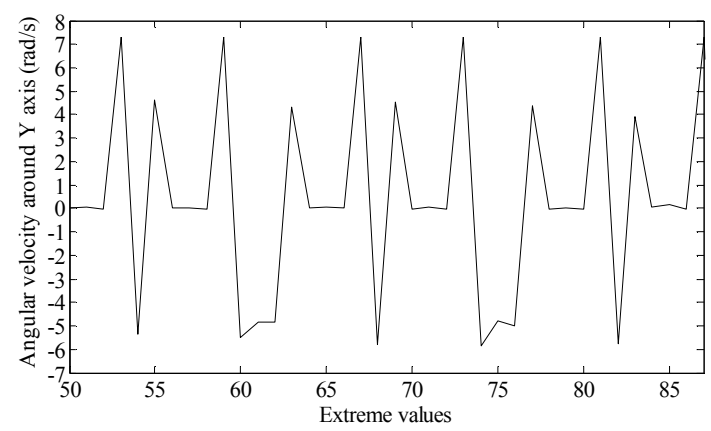

c) Extreme points of angular velocity around $\mathrm{Y}$ axis

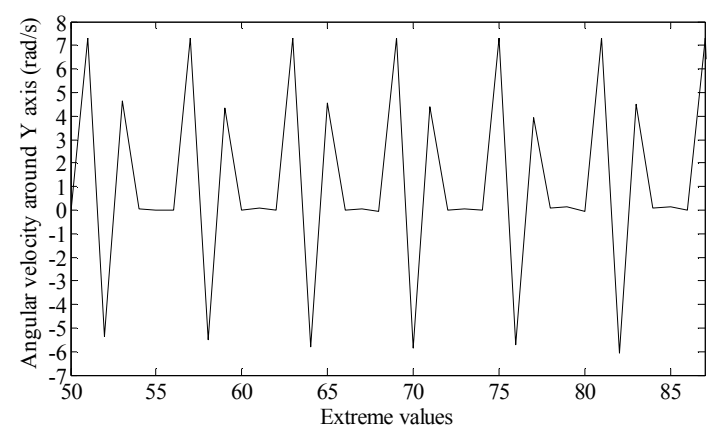

d) Removed redundant extreme in state $B$ 


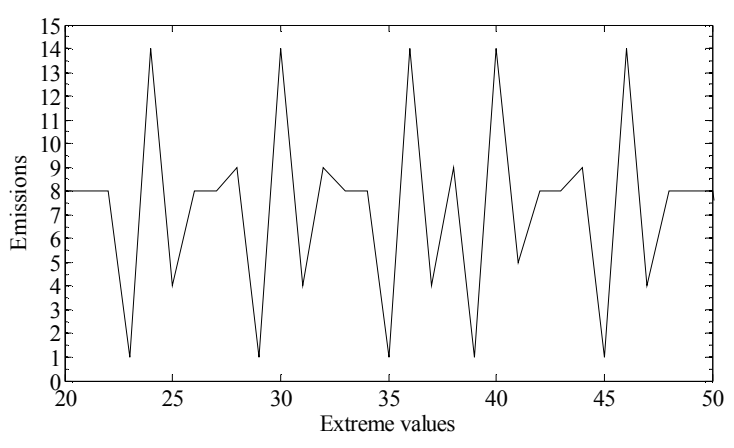

e) Observations of angular velocity around $\mathrm{Y}$ axis

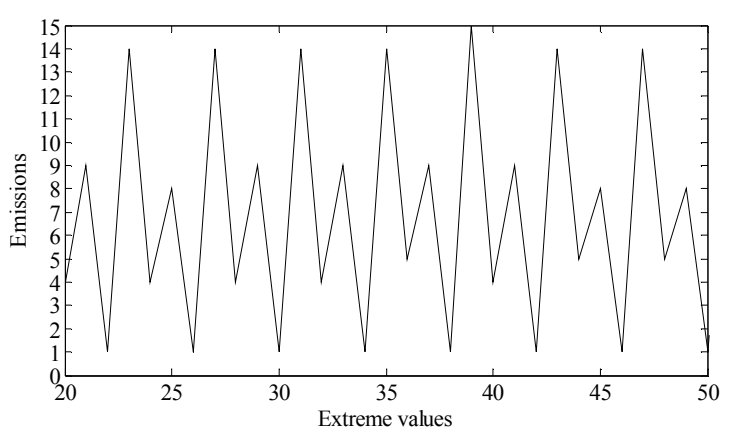

f) Removed redundant extreme in state D.

Fig.5. The flow chart of observations extraction algorithm.

Table 1. Statistical analysis results of observation.

\begin{tabular}{|c|c|c|c|c|}
\hline \multirow{2}{*}{ Observation } & \multicolumn{5}{|c|}{ Probability (\%) } \\
\cline { 2 - 5 } & State A & State B & State C & State D \\
\hline 1 & 72.3 & 12.3 & 0 & 0 \\
\hline 2 & 9.23 & 1.54 & 0 & 1.52 \\
\hline 3 & 1.54 & 0 & 7.69 & 1.52 \\
\hline 4 & 0 & 0 & 43.08 & 7.58 \\
\hline 5 & 0 & 0 & 30.77 & 4.55 \\
\hline 6 & 1.54 & 0 & 1.54 & 0 \\
\hline 7 & 0 & 1.54 & 0 & 0 \\
\hline 8 & 7.69 & 0 & 0 & 24.2 \\
\hline 9 & 7.69 & 0 & 0 & 60.6 \\
\hline 10 & 0 & 0 & 1.54 & 0 \\
\hline 11 & 0 & 3.08 & 0 & 0 \\
\hline 12 & 0 & 1.54 & 1.54 & 0 \\
\hline 13 & 0 & 1.54 & 1.54 & 0 \\
\hline 14 & 0 & 73.85 & 12.31 & 0 \\
\hline 15 & 0 & 6.15 & 0 & 0 \\
\hline
\end{tabular}

The algorithm is introduced in detail as follows: at first, raw angular velocity around the $\mathrm{Y}$ axis is stored in a vector (see Fig.5.a)); and then in order to eliminate small noise values, the vector is smoothed using moving average method (see Fig.5.b)) after that, the maximum and minimum values from the vector are used to describe each state (see Fig.5.c)). From experimental results, we find that consecutive extreme values exist within state $\mathrm{B}$ and $\mathrm{D}$. Because consecutive extreme values stand for the same state, so redundant extreme values are deleted (see Fig.5.d) and f)); then, the observation value is used to present the processed $\mathrm{Y}$ axis angular velocity according to 15 grades (see Fig.4. and Fig.5.e)). As mentioned above, observations from raw angular velocity are acquired and the data volume is only $5 \%$ of raw data volume. The result obtained through the analysis of observation vector is shown in Table 1 .

\subsection{Estimated probability optimization}

As mentioned above, state transition probability and observation probability have been initialized. In order to optimize the model parameter, the Baum-Welch algorithm and the sample set were used to train the model parameter. The adopted sample set (data volume is 55459) was collected in an experiment. The Baum-Welch algorithm is a particular case of a generalized expectation-maximization algorithm. It can compute the maximum likelihood estimate and posterior mode estimates for the parameters (transition and observation probabilities) of an HMM. The algorithm process is as follows [17]:

$$
\begin{gathered}
\overline{a_{i j}}=\frac{\sum_{t=1}^{T} \alpha_{t-1}(i) \alpha_{i j} b_{j}\left(O_{t}\right) \beta_{t}(j)}{\sum_{t=1}^{T} \alpha_{t-1}(i) \beta_{t-1}(i)} \\
\overline{\pi_{i}}=\frac{\alpha_{1}(i) \beta_{1}(i)}{\sum_{j=1}^{N} \alpha_{T}(j)}
\end{gathered}
$$

$$
\bar{c}_{j m}=\frac{\sum_{t=1}^{T} \gamma_{t}(j, m)}{\sum_{t=1}^{T} \sum_{m=1}^{M} \gamma_{t}(j, m)}
$$

$$
\bar{\mu}_{j m}=\frac{\sum_{t=1}^{T} \gamma_{t}(j, m) O_{t}}{\sum_{t=1}^{T} \gamma_{t}(j, m)}
$$

$$
\bar{U}_{j m}=\frac{\sum_{t=1}^{T} \gamma_{t}(j, m) \llbracket\left(O_{t}-\mu_{j m}\right)\left(O_{t}-\mu_{j m}\right)}{\sum_{t=1}^{T} \gamma_{t}(j, m)}
$$

Where $\bar{\pi}_{i}, \bar{a}_{i j}, \bar{c}_{j m}, \bar{u}_{j m}$ and $\bar{U}_{j m}$ are the model parameters, $\gamma_{t}(j, m)$ is the probability of beginning in state $j$ at time $t$ with $m_{t h}$ mixture component accounting for $O_{t}$ of the form

$$
\gamma_{t}(j, m)=\left[\frac{\alpha_{t}(i) \beta_{t}(i)}{\sum_{i=1}^{N} \alpha_{t}(j) \beta_{t}(i)}\right]\left[\frac{c_{j m} G\left(O_{t}, \mu_{j m}, U_{j m}\right)}{\sum_{k=1}^{M} c_{j k} G\left(O_{t}, \mu_{j k}, U_{j k}\right)}\right]
$$

Optimized state transition probability and observation probability are as follows, and the state transition diagram is shown in Fig.6. The optimized observation probability is shown in Table 2.

$$
A=\left[\begin{array}{cccc}
0 & 0.9620 & 0.0247 & 0.0133 \\
0.0110 & 0.0310 & 0.9253 & 0.0326 \\
0.0016 & 0.0135 & 0.0344 & 0.9505 \\
0.9717 & 0.0115 & 0.0166 & 0
\end{array}\right]
$$




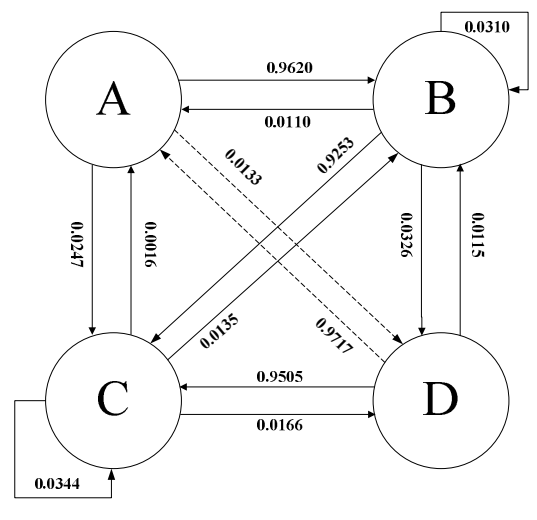

Fig.6. The state transition diagram.

Table 2. Optimized observation probability.

\begin{tabular}{|c|c|c|c|c|}
\hline \multirow{2}{*}{ Observation } & \multicolumn{5}{|c|}{ Probability (\%) } \\
\cline { 2 - 5 } & State A & State B & State C & State D \\
\hline 1 & 78.09 & 0 & 0 & 0 \\
\hline 2 & 16.98 & 0 & 0 & 0 \\
\hline 3 & 2.83 & 0 & 17.29 & 0 \\
\hline 4 & 0 & 0 & 46.63 & 0 \\
\hline 5 & 0 & 0 & 27.06 & 0 \\
\hline 6 & 2.11 & 0 & 5.27 & 0 \\
\hline 7 & 0 & 3.1 & 0 & 0 \\
\hline 8 & 0 & 0 & 0 & 38.95 \\
\hline 9 & 0 & 0 & 0 & 61.05 \\
\hline 10 & 0 & 0 & 0.49 & 0 \\
\hline 11 & 0 & 3.75 & 0 & 0 \\
\hline 12 & 0 & 0 & 3.26 & 0 \\
\hline 13 & 0 & 16.15 & 0 & 0 \\
\hline 14 & 0 & 68.19 & 0 & 0 \\
\hline 15 & 0 & 8.81 & 0 & 0 \\
\hline
\end{tabular}

It is important to note that optimized state transition probability and observation probability are based on raw angular velocity measured by MEMS inertial gyroscopes, so it relates to specific experimental parameters, such as the walking characteristics of experimenters and different experimental environment. Therefore, the optimized state transition probability and observation probability (obtained after applying the Baum-Welch algorithm) are not used for all the datasets, and the optimized state transition probability and observation probability should be optimized with different databases, just as the train process.

\section{PEDESTRIAN NAVIGATION SYSTEM FRAMEWORK}

Today, pedestrian navigation system widely adopts a system framework that is characterized by extended Kalman filtering and strapping MEMS inertial sensors on insteps, which is proposed by Eric Foxlin, whereby the filter tracks the errors in the system state rather than the system state directly, named an error-state, or complementary filter. In addition to the values of state errors, the filter also estimates their error covariance and cross-covariance, which enables the filter to correct the position (not only the velocity) during a ZUPT. As a pseudo-measurement, zero velocity provides the required information to reset the state errors when the IMU is detected in stationary period between each footstep. During the normal walking, zero-velocity occurs during the stance phase, when one foot is carrying the full weight of the body, which has made the solution based on foot-mounted IMU a popular choice for the PIN system.

In our system, a novel zero velocity detection algorithm is used, and the framework is shown in Fig.7. As mentioned above, the angular velocity around the $\mathrm{Y}$ axis is used to detect zero velocity. The navigation software based on this framework is developed and will be introduced in the next section.

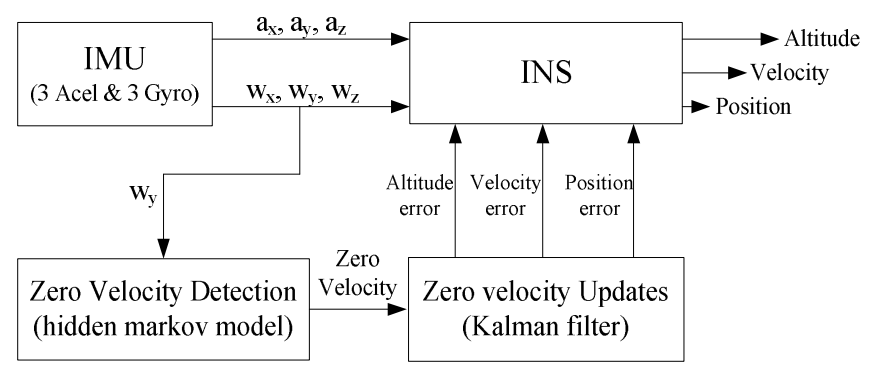

Fig.7. The pedestrian navigation system framework diagram.

\section{Pedestrian NAVIGATION FIELD TEST}

In this section, the navigation software based on MATLAB platform is described; secondly, the field test is introduced; finally, a general description of the experiments is given.

\subsection{Navigation software}

The experiments were run using custom software developed based on the MATLAB platform, which processes the data measured by inertial sensors in off-line way. The system is built on the extended Kalman filtering framework mentioned above. In addition, the relevant parameters, such as distance travelled, and displacement in 3D space, are computed accurately. Finally, the trajectory of a pedestrian can be plotted in vertical view, side view, and stereo view. It is worth mentioning that the animation is used to perform the movement process.

The user interface of the pedestrian navigation software is shown in Fig.8.

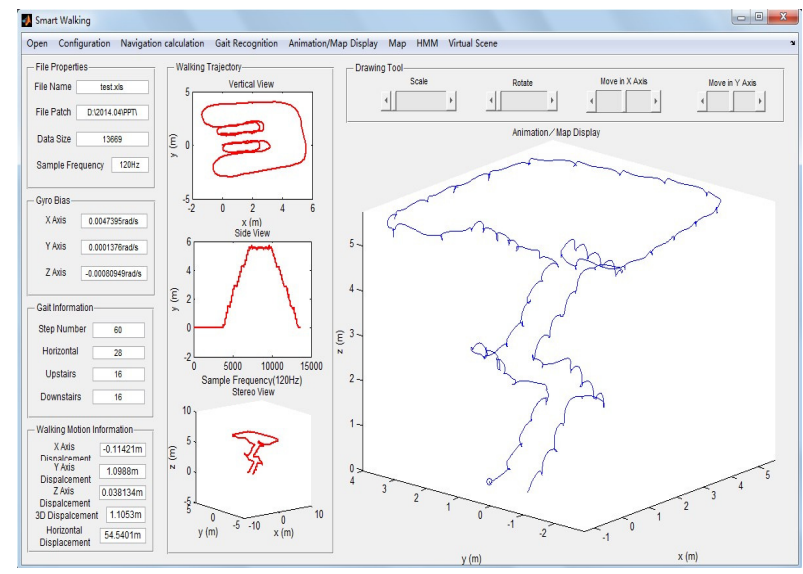

Fig.8. The User interface of navigation software. 


\subsection{Field tests}

In order to verify the validity of our navigation system, several field tests have been conducted. Generally, the field test can be divided into two kinds according to the navigation environments, one is outdoor test, and the other is indoor test. A square is selected as outdoor field, and a lab building with complex interior structure is selected as indoor field. The lab building has five floors, as a general building, each floor is connected by stairs, and an interlayer exists between two floors.

The experimenter is a male who is $1.80 \mathrm{~m}$ tall and weighs $65 \mathrm{~kg}$. In addition, the walking speed is $1.5 \mathrm{~m} / \mathrm{s}$ on average.

As mentioned above, the inertial sensor used is MTx (MTx-28A53G25) from Xsens technologies B.V., which is attached with shoelaces on the instep of the pedestrian.

\subsubsection{Outdoor test}

The outdoor test was conducted on a square, and the planned path was along the sidelines of a square, which was a $3925 \mathrm{~m}^{2}$ circular area and the radius was $50 \mathrm{~m}$, as shown in Fig.9. More specifically, two tests have been conducted on this square, one is rectangle walking motion which is plotted in red, and the other is circular walking motion which is plotted in blue.

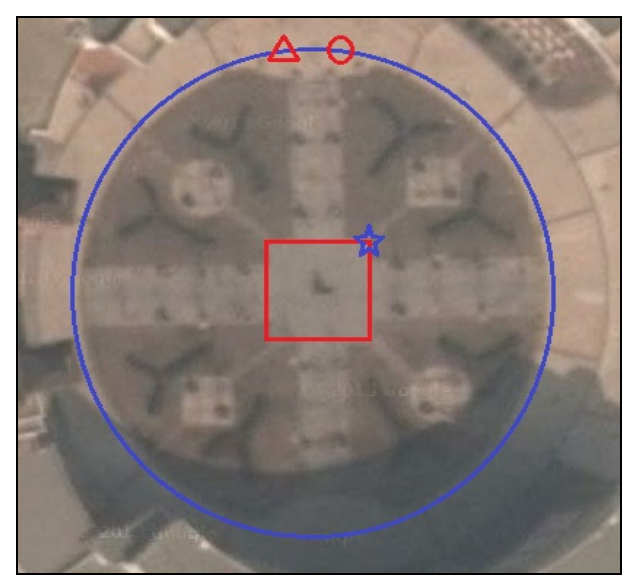

Fig.9. The satellite photo of the test square.

To begin with, the rectangle walking motion is analyzed. The perimeter is $75.00 \mathrm{~m}$ measured by the experimenter, and the path length is $74.39 \mathrm{~m}$ computed by the navigation system. The trajectory is plotted in vertical view as shown in Fig.10. As the route is closed, the start point and the end point are overlapping in theory, but the reality is different, as shown in Fig.10. and in Table 2. The displacement error is $2.3 \mathrm{~m}, 3.0 \%$ of total path length.

The second test is a circular walking test, and the planned path is plotted in blue as shown in Fig.9. The triangle symbol is the starting point, walking in the counter-clockwise direction until reaching the circular symbol, which is the end point. As mentioned above, the actual measured distance of walking path is $304 \mathrm{~m}$, as $10 \mathrm{~m}$ distance exists between starting and end point. The path length is $310 \mathrm{~m}$ computed by the navigation system. The trajectory is plotted in vertical view as shown in Fig. 11. The displacement error is $6 \mathrm{~m}, 2 \%$ of total path length.

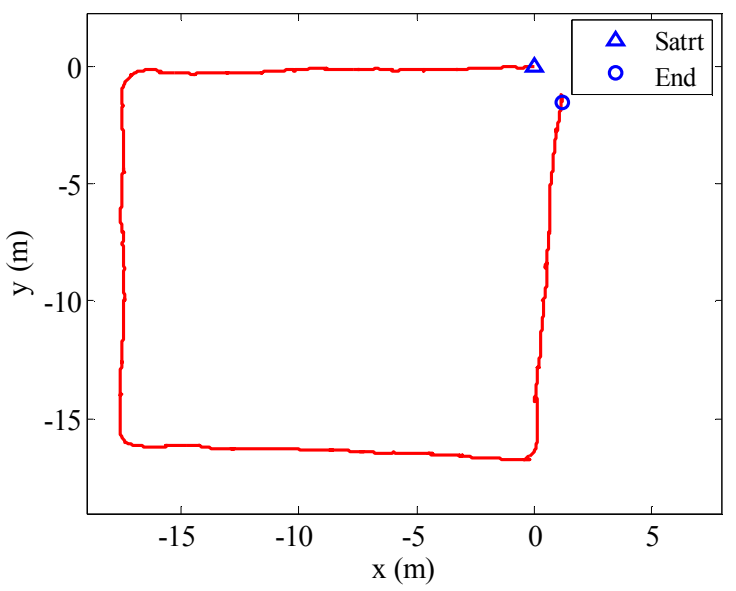

Fig.10. The walking trajectory of rectangle walking motion.

Table 3. Navigation error of rectangle walking motion.

\begin{tabular}{|c|c|}
\hline Direction & Displacement [m] \\
\hline X Axis & 1.20 \\
\hline Y Axis & 1.50 \\
\hline
\end{tabular}

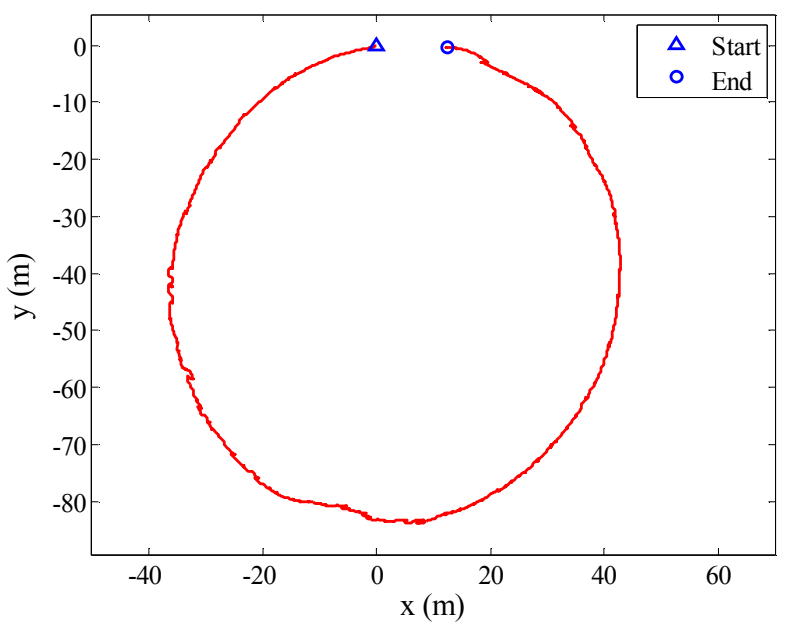

Fig.11. The walking trajectory of circular walking motion.

Table 4. Navigation error of circular walking motion.

\begin{tabular}{|c|c|}
\hline Direction & Displacement $[\mathbf{m}]$ \\
\hline X Axis & 12.25 \\
\hline Y Axis & 0.4 \\
\hline
\end{tabular}

As mentioned above, the two outdoor test results show that accuracy of pedestrian navigation system is reliable, about $2 \sim 3 \%$ of total path length. Nevertheless, the outdoor tests are conducted on the ground, in order to research the navigation accuracy in buildings; the indoor tests are conducted, which will be discussed in the next section.

\subsubsection{Indoor test}

The indoor tests are conducted in a lab building with complex interior structure. The lab building which has five floors, as a general building, each floor is connected by stairs, and an interlayer exists between two floors. 
To begin with, the first test is analyzed, the walking planned path is a closed route from a point on NO.1 floor, then going upstairs to NO.2 floor, through the corridors, and going downstairs back to NO.1 floor, finally going back to the starting point. As the route is closed, the starting point and the end point are overlapping in theory, but the reality is different, as shown in Fig.12., which is a $3 \mathrm{D}$ view of the walking trajectory. In addition, the navigation error is shown in Table 4. And the height walking trajectory is shown in Fig.13. The displacement error is $1.95 \mathrm{~m}, 2.4 \%$ of total path length.

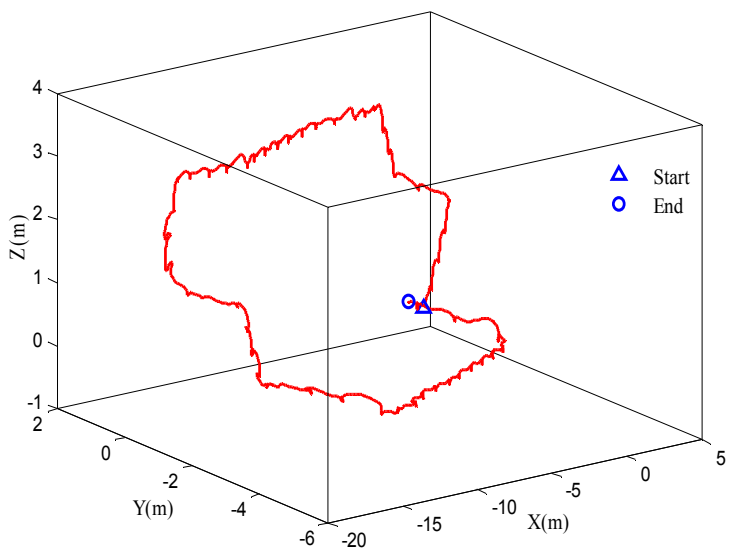

Fig.12. The 3D walking trajectory.

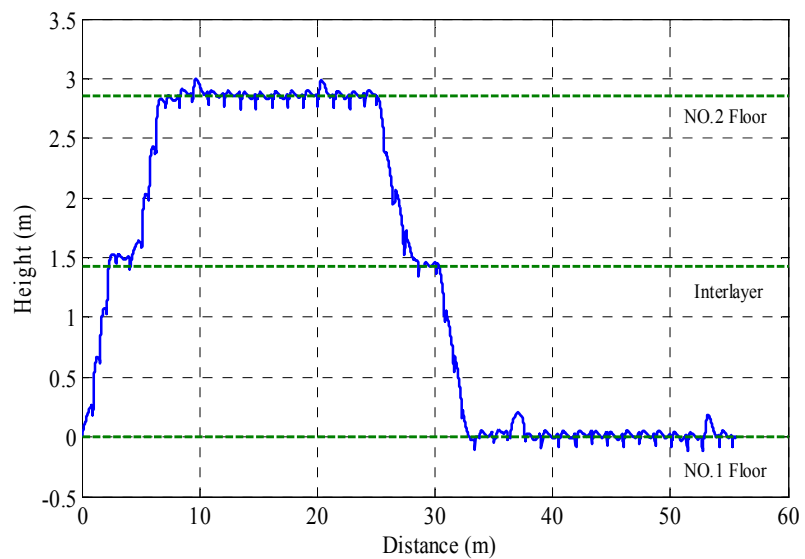

Fig.13. The height walking trajectory.

Table 5. Navigation error of first indoor test.

\begin{tabular}{|c|c|}
\hline Direction & Displacement [m] \\
\hline X Axis & 1.25 \\
\hline Y Axis & 1.50 \\
\hline Z Axis & 0.10 \\
\hline
\end{tabular}

Secondly, another indoor test in the lab building was conducted, specifically, the planned walking path covered three floors, and the starting point was on NO.1 floor, climbing up to NO.3 floor and a rectangular path was followed on this floor, then climbing down to NO.1 floor through another flight of stairs. The end point deviates from the start point in the $\mathrm{Y}$ axis about $1.0 \mathrm{~m}$.

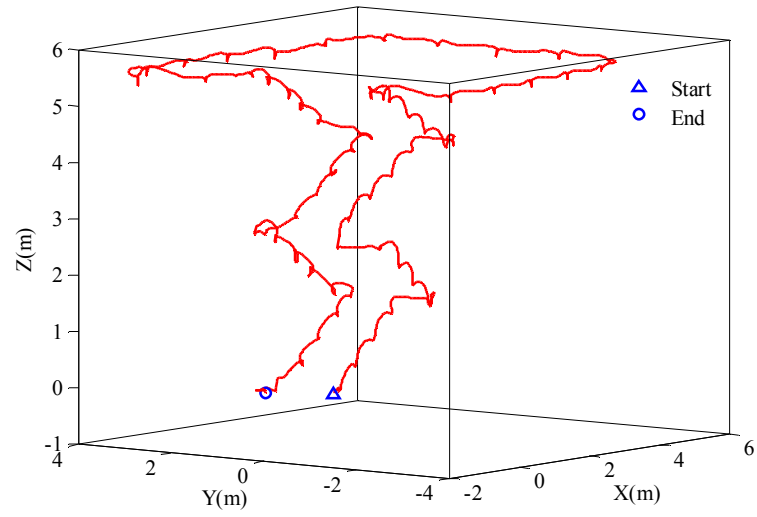

Fig.14. The 3D walking trajectory.

The computed 3D walking trajectory is shown in Fig.14., and the height walking trajectory is shown in Fig.15. The navigation error is shown in Table 4 . The displacement error is $1.80 \mathrm{~m}, 1 \%$ of total path length.

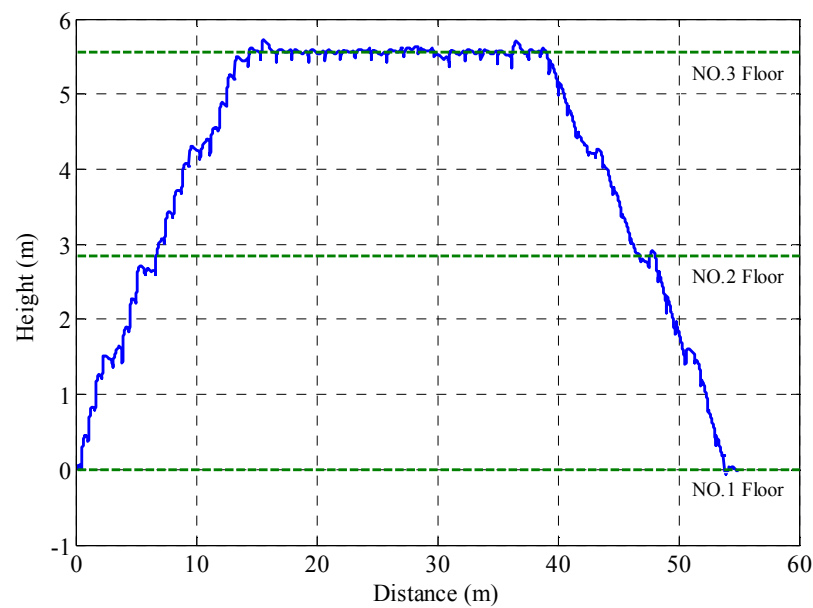

Fig.15. The height walking trajectory.

Table 6. Navigation error of second indoor test.

\begin{tabular}{|c|c|}
\hline Direction & Displacement [m] \\
\hline X Axis & 1.32 \\
\hline Y Axis & 1.22 \\
\hline Z Axis & 0.15 \\
\hline
\end{tabular}

As mentioned above, two outdoor navigation tests and two indoor navigation tests were conducted, which were processed with the pedestrian navigation software developed on MATLAB. The computed displacement in three axes was analyzed, the navigation error of outdoor and indoor navigation field tests was less than $3 \%$ of total distance travelled. The walking trajectories were plotted in different view. The experimental results indicate that the pedestrian navigation system proposed in this paper can eliminate error caused by inertial sensors and other factors effectively, and the navigation algorithm is suitable for horizontal navigation and stereo navigation, and the navigation accuracy is acceptable. 


\section{CONCLUSIONS}

Zero velocity detection is an essential part of pedestrian inertial system, it provides the required information to reset velocity estimated error, otherwise the velocity estimated error would increase linearly with time, and the estimated position error would increase at least quadratically. In this paper, in order to detect zero velocity state accurately, a zero velocity detection algorithm based on the hidden Markov model is proposed. The core idea is describing zero velocity detection with the hidden Markov model, and four states are used to describe the walking motion. Therefore, zero velocity detection is converted to detect a state in the hidden Markov model using the Baum-Welch algorithm. In addition, pedestrian navigation software was developed based on the extended Kalman filtering and strapping MEMS inertial sensors on insteps. Finally, employing the several indoor and outdoor navigation tests in lab buildings and squares, we evaluated the performance of pedestrian inertial navigation based on hidden Markov model proposed in this paper. The test results show that the position error was less than $3 \%$ of total distance travelled. In addition, it works well in indoor and outdoor navigation environment.

\section{ACKNOWLEDGMENT}

This work was supported by the National Natural Science Foundation Projects of China (NO.61073134, NO.51179020, NO.51309044); the Fundamental Research Funds for the Central Universities (NO.3132014027, NO.3132014203, NO. 3132013319, NO.3132013014, and NO.3132013017); the Applied Fundamental Research Project of Ministry of Transport of China (NO.2013329225290); the Scientific Research Project of Liaoning Education Department (NO.L2013208).

\section{REFERENCES}

[1] Dedes, G., Dempster, A.G. (2005). Indoor GPS positioning-challenges and opportunities. In 62nd Vehicular Technology Conference (VTC-2005 -Fall), 25-28 September 2005. IEEE, 412-415.

[2] Jim Nez, A.R., Seco, F., Zampella, F., Prieto, J.C., Guevara, J. (2011). PDR with a foot-mounted IMU and ramp detection. Sensors, 11 (10), 9393-9410.

[3] Ali, J. (2009). Astronavigation system as an autonomous enhancement suite for a strapdown inertial navigation system: An evaluation. Measurement Science Review, 9 (2), 42-48.

[4] Harle, R. (2013). A survey of indoor inertial positioning systems for pedestrians. IEEE Communications Surveys \& Tutorials, 15 (3), 1281-1293.

[5] Foxlin, E. (2005), Pedestrian tracking with shoe-mounted inertial sensors. IEEE Computer Graphics and Applications, 25 (6), 38-46.

[6] Fischer, C., Talkad Sukumar, P., Hazas, M. (2013), Tutorial: Implementing a pedestrian tracker using inertial sensors. IEEE Pervasive Computing, 12 (2), 17-27.
[7] Wen, L., Yingjun, Z., Feixiang, Z. (2013). A gait recognition algorithm using MEMS inertial sensor for pedestrian dead-reckoning. In 20th Saint Petersburg International Conference on Integrated Navigation Systems, 27-29 May 2013, 284-287.

[8] Park, S.K., Suh, Y.S. (2010). A zero velocity detection algorithm using inertial sensors for pedestrian navigation systems. Sensors, 10 (10), 9163-9178.

[9] Yun, X., Bachmann, E.R., Moore, H., Calusdian, J. (2007). Self-contained position tracking of human movement using small inertial/magnetic sensor modules. In IEEE International Conference on Robotics and Automation, 10-14 April 2007. IEEE, 2526-2533.

[10] Ojeda, L., Borenstein, J. (2007). Non-GPS navigation with the personal dead-reckoning system. In Unmanned Systems Technology IX, 9-12 April 2007. SPIE, Vol. 6561.

[11] Bousbia-Salah, M., Fezari, M. (2006). The development of a pedestrian navigation aid for the blind. In IEEE GCC Conference, 20-22 March 2006. IEEE, 1-5.

[12] Godha, S., Lachapelle, G., Cannon, M.E. (2006). Integrated GPS/INS system for pedestrian navigation in a signal degraded environment. In 19th International Technical Meeting of the Satellite Division (ION GNSS 2006), 26-29 September 2006. Fort Worth, TX, 2151-2164.

[13] Jimenez, A.R., Seco, F., Prieto, J.C., Guevara, J. (2010). Indoor pedestrian navigation using an INS/EKF framework for yaw drift reduction and a foot-mounted IMU. In 7th Workshop on Positioning Navigation and Communication, 11-12 March 2010. IEEE, 135-143.

[14] Skog, I., Nilsson, J.O., Handel, P. (2010). Evaluation of zero-velocity detectors for foot-mounted inertial navigation systems. In International Conference on Indoor Positioning and Indoor Navigation, 15-17 September. IEEE, 1-6.

[15] Wen, L., Yingjun, Z. (2013). Feature analysis of pedestrian navigation using energy-saving inertial sensors. Energy Education Science and Technology Part A: Energy Science and Research, 31 (1), 461-464.

[16] Cheshomi, S., Rahati-Q, S., Akbarzadeh-T, M.-R. (2010). Hybrid of Chaos Optimization and Baum-Welch algorithms for HMM training in continuous speech recognition. In International Conference on Intelligent Control and Information Processing, 13-15 August 2010. IEEE, 83-87.

[17] Rabiner, L. (1989). A tutorial on hidden Markov models and selected applications in speech recognition. Proceedings of the IEEE, 77 (2), 257-286.

Received July 4, 2014. Accepted February 25, 2015. 